\title{
High-Pressure Xenon Detector in Nuclear Radiation Measurement System
}

\author{
Xinyang Fan, Tibo Yang \\ School of Nuclear Technology and Automation Engineering, Chengdu University of Technology, Chengdu, China \\ Email:2711846131@qq.com
}

How to cite this paper: Fan, X.Y. and Yang, T.B. (2020) High-Pressure Xenon Detector in Nuclear Radiation Measurement System. Open Access Library Journal, 7: e6690.

https://doi.org/10.4236/oalib.1106690

Received: August 5, 2020

Accepted: August 21, 2020

Published: August 24, 2020

Copyright $\odot 2020$ by author(s) and Open Access Library Inc.

This work is licensed under the Creative Commons Attribution International License (CC BY 4.0).

http://creativecommons.org/licenses/by/4.0/

\section{(c) (i) Open Access}

\begin{abstract}
With its excellent energy resolution and good temperature characteristics, high-pressure xenon detectors are widely used in extreme measurement environments such as high temperature and strong radiation. However, the energy resolution of the energy spectrum of the high-pressure xenon detector will be reduced or even distorted in a high-decibel noise environment. In order to better understand the noise sensitivity of high-pressure xenon detectors, this paper summarizes and analyzes the principle, development process and research status of high-pressure xenon detectors based on the literature on high-pressure xenon detector structure and performance at home and abroad. The study found that for the problem of baseline drift caused by the noise sensitivity of high-pressure xenon detectors, the traditional baseline restoration algorithm cannot effectively play a role. The adaptive baseline restoration method should be able to overcome this problem.
\end{abstract}

\section{Subject Areas}

Nuclear Physics

\section{Keywords}

High-Pressure Xenon Detector, Nuclear Radiation Measurement, Energy Resolution

\section{Introduction}

In nuclear physics research, high-energy resolution radiation monitoring is required for complex environments such as high temperatures and strong magnetic fields [1]. Semiconductor detectors have the advantages of high energy resolution, wide energy linear range, fast output pulse rise time, and small size [2], but semiconductor detectors have reverse currents, which are difficult to use at 
room temperature or environments with large temperature fluctuations [3] and large-scale crystal growth is also a problem for semiconductor detectors [4]. Scintillator detectors can detect radiation in complex environments due to their advantages in volume and temperature performance, but insufficient energy resolution has always been a defect of scintillator detectors [5]. The high-pressure xenon detector is a high-energy resolution gamma gas detector with good physical characteristics. It has a wide operating temperature range, excellent anti-irradiation ability and working life, and is suitable for complex radiation detection environments [6]. Although the high-pressure xenon detector has many performance indicators in nuclear radiation measurement, the grid vibration caused by its noise can cause serious defects such as distortion of energy resolution. The traditional high-pressure xenon detector uses analog multi-channel technology for energy spectrum measurement, there are a large number of discrete components, the design is too complicated, and the simulated multi-channel is susceptible to external noise interference, which cannot solve the problem of baseline drift caused by noise and vibration [7]. With the development of digital signal processing technology, digital multi-channel technology and digital pulse synthesis technology have been widely used in nuclear radiation measurement systems [8], which provides a technical basis for solving High-pressure xenon's own defects.

In view of the above characteristics, based on the research of high-pressure xenon detectors by domestic and foreign researchers, this article first explains the principles of high-pressure xenon detectors, and describes in detail the development process of high-pressure xenon detectors in the nuclear energy spectrum measurement system and the current research status, and then summarizes the report, conducts a systematic analysis of digital multi-channel technology, and finally gives my own views and prospects. These works provide a comprehensive reference for the subsequent improvement of the temperature of the high-pressure xenon detector's energy spectrum measurement and the improvement of the energy resolution.

\section{Principle of High-Pressure Xenon Detector}

The high-pressure xenon detector is a screen-grid ionization chamber, which belongs to the range of flat-panel ionization chambers. The working principle of the ionization chamber is to construct an electric field in the sensitive volume between the two electrodes of the ionization chamber. Its strength is sufficient to avoid the occurrence of ion pair recombination. The incident charged particles ionize in the sensitive volume to form ion pairs. The ion pairs are under the action of the electric field. Produce directional drift, thus output current signal in the loop [9]. Figure 1 is a schematic diagram of a typical flat ionization chamber [10].

In Figure 1(a), $x$ is the position where the rays interact with the substance, $d$ is the spacing of the entire flat-panel ionization chamber, $C$ is the detector capa- 
citance, and $R$ is the external load resistance. In Figure $1(\mathrm{~b}), t$ is the time for electrons to drift to the anode, and $t^{+}$is the time for ions to drift to the anode. Usually the drift speed of electrons is much larger than that of ions, so $t^{+}$is much greater than $t$ [11].

Figure $1(\mathrm{~b})$ is a reference diagram of the pulse shape corresponding to various RC for the flat-plate ionization chamber. It can be seen from the figure that under different RC value conditions, different output voltage pulse signals will be formed, and completely different working states of the ionization chamber will be formed. It can be seen from Figure 1(b) that if the decay time constant RC is much larger than the drift velocity of the ion, the charge will be completely collected and there will be no charge loss. In fact, if a very long time constant is maintained, the capacitor cannot maintain a stable amount of electricity, and too large a decay time constant will reduce the pulse pass rate, so this method is not used. When performing energy spectrum measurement, usually choose a time constant that is longer than the electron collection time and shorter than the ion collection time. The pulse voltage generated at this time is Equation (1):

$$
V=\frac{n_{0} e}{C} \times \frac{x}{d}
$$

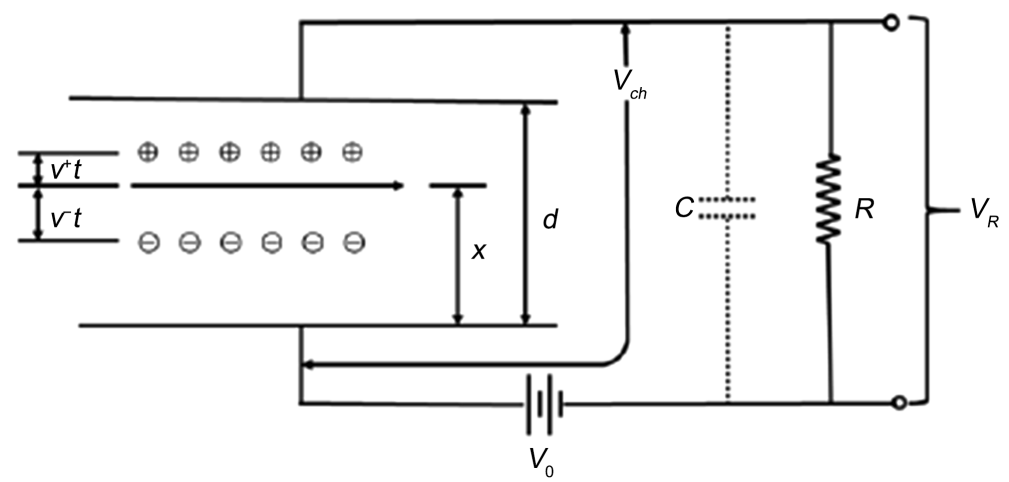

(a)

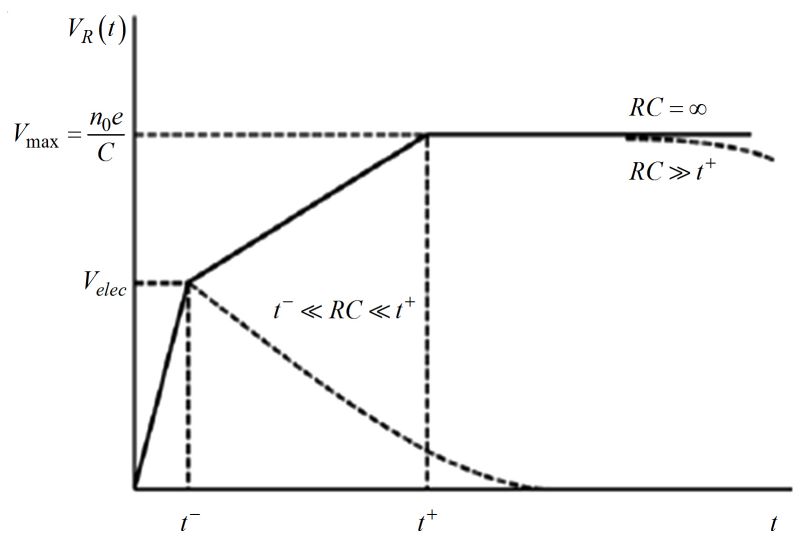

(b)

Figure 1. The structure of the flat panel ionization chamber and the output pulse shape [10]. (a) Schematic diagram of flat ionization chamber; (b) Pulse shape under different time constants (The unit of $t$ is $\mathrm{ms}$ ). 
It can be seen from Equation (1) that the generated pulse amplitude is related to the ionization position of the radiation in the ionization chamber. Since the probability of interaction between radiation and matter is random, the amplitude obtained by Equation (1) is not a linear value. In order to solve this problem, the high-pressure xenon detector has a grid electrode with parallel filaments between the anode and cathode of the ordinary flat ionization chamber, called Frisch, and its potential is between the anode and the cathode. The structure diagram of the High-pressure xenon detector is shown in Figure 2 [11].

By using a collimator or determining a suitable position for the radiation source, the interaction between the incident charged particles and the working gas is strictly limited between the cathode and the Frisch grid. The generated positive ions directly float to the cathode under the action of the electric field, and the electrons float to the grid and continue to float to the anode through the grid. While the Frisch grid plays a shielding role, it should be transparent to electrons so that it can pass smoothly. The pulse voltage value obtained in this way is shown in formula (2):

$$
V=\frac{n_{0} e}{d C} v^{-t}
$$

In formula (2), $d$ is the distance between the grid of the screen grid ionization chamber and the anode. As time changes, the voltage increases linearly until the electrons reach the anode. The signal voltage is the largest, that is, $d=v^{-t}$, and the pulse amplitude is formula (3):

$$
V=\frac{n_{0} e}{C}=\frac{E}{W} \cdot \frac{e}{C}
$$

In formula (3), $n_{0}$ is the number of charges, $e$ is the amount of charge, $C$ is the energy of the incident particles, and $W$ is the average ionization energy. It can be seen from equation (3) that the generated pulse radiation has nothing to do with the initial ionization position, and is proportional to the incident particle energy.

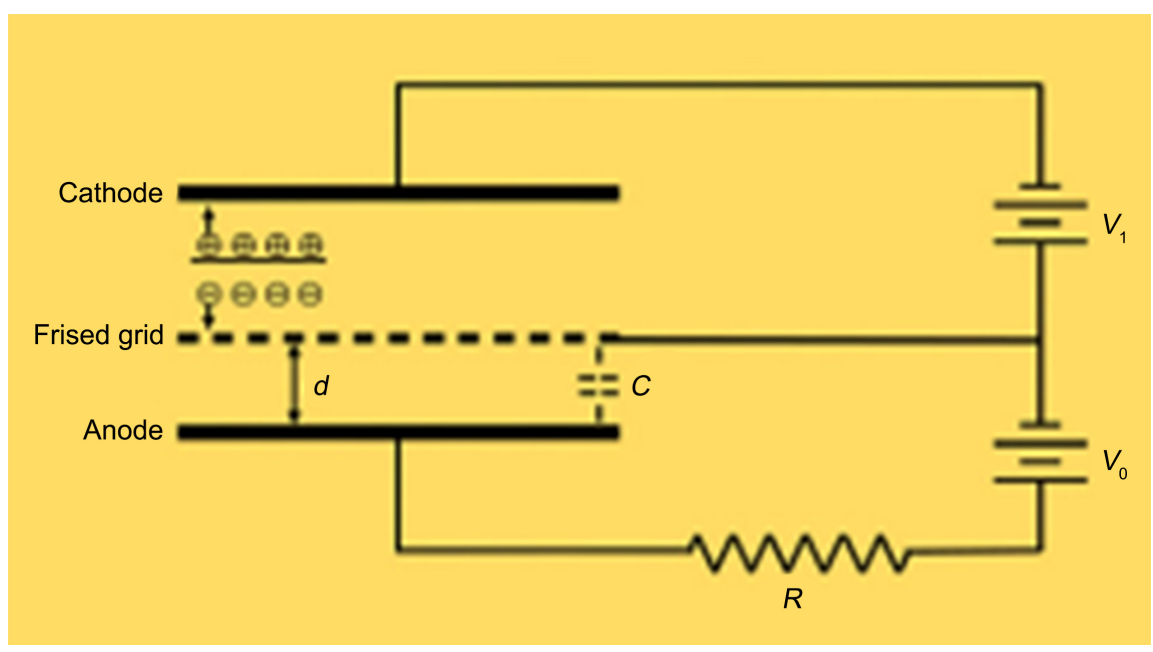

Figure 2. Structure diagram of High-pressure xenon detector [11]. 


\section{Development of High-Pressure Xenon Detector}

In the 1940s, Davidson [12] and Hutchinson [13] added liquid inert gas to the ionization chamber for the first time and discovered the conductivity of liquid inert gas under ionizing radiation. In the 1980s, researchers theoretically predicted that the energy resolution of liquid xenon detectors could reach $0.4 \%$ (1 $\mathrm{MeV}$ ) [14], but limited to the processing technology at the time, the actual energy resolution of liquid xenon detectors could not exceed 5\% [15], a huge gap with theory. In order to improve the energy resolution of the detector, Russian engineering researchers began to try to replace liquid xenon with gaseous xenon, and in the late 1980s they studied high-pressure xenon detectors for gamma energy measurement [16]. In the 1990s, with the rapid development of materials science, it was discovered that the cylindrical [17] and flat-type [18] high-voltage xenon ionization chambers using Frisch screen grid technology had an energy resolution of $2 \%$ to $3 \%$. Since then, people began to conduct a lot of experimental research on the screen grid structure of Frisch. Dmitrenko et al. found that the high-voltage xenon ionization chamber of the screen grid structure is more severely affected by external noise and vibration, which can seriously cause energy resolution distortion [19].

The impact of noise and vibration of high-pressure xenon detectors has become a hot spot in the research of high-pressure xenon detectors. The screen grid structure of the high-pressure xenon detector is very thin, and small external vibrations will cause its structure to change, resulting in poor energy resolution. Kessick, Boolotnikov and others began to study the structure of a new high-voltage xenon detector ionization chamber that changed the screen grid structure, and developed a half-type ionization chamber [20], a double anode cylindrical ionization chamber [21], and a common anode cylindrical ionization chamber [22]. Zeng Yong of Sichuan University designed a high-voltage xenon ionization chamber with a screen grid array structure, and used Monte Carlo method to conduct theoretical research on its performance [5]. Wang Wei, a researcher at the Chinese Academy of Atomic Energy, used the Maxwell3D program to calculate the influence of the number and position of the pole filaments on the energy resolution of a high-voltage xenon ionization chamber with a coplanar anode structure [23]. Although these ionization chambers with different structures solve the problem of energy resolution distortion caused by screen grid vibration, their energy resolution is far inferior to the Frisch screen grid high-pressure xenon detector. Therefore, this problem cannot be solved well by changing the structure of the ionization chamber. Later, people returned the focus of research to the high-pressure xenon detector with the screen grid structure. The researchers tried to wrap noise-absorbing shielding materials outside the Frisch screen grid structure high-pressure xenon detector. The experimental results found that although the noise effect is small, the volume and weight of the detector are increased. Dmetrenko et al. carried out research on the screen grid structure material [24], by increasing the thickness and strength of the 
screen grid material to improve the impact on external noise, the experimental results show that the stability of the energy resolution can be maintained within a certain vibration range.

\section{Research Status of High-Pressure Xenon Detector}

While the Frisch screen grid of the high-pressure xenon detector improves the energy resolution of the energy spectrum, it has been perplexing researchers due to its own screen grid sensitivity. In a noisy environment, High-pressure xenon detectors will have serious baseline wandering problems.

Figure 3 is a schematic diagram of the cylindrical high-pressure xenon screen grid ionization chamber [11]. The signal is read from the anode filament in the center. The anode is opposite to the grid, and the middle is filled with high-voltage xenon gas to form a cylindrical capacitor with xenon gas as the insulating medium and the anode and grid as two electrodes. The two poles of the capacitor are fixed with high voltage. Due to the large area and thin thickness of the Frisch grid, when the detector is in a vibration and noise environment, the vibration and noise will cause the grid to move. The shift of the grid position causes the capacitance between the two poles to change, and finally makes the detector output pulse baseline Offset occurred.

With the development of digital signal processing technology, digital signal processing methods with programmability and flexibility are widely used in the processing of complex signals [25]. The continuous maturity of high-speed ADC and resource-rich programmable gate array logic device technology FPGA, digital filtering technology and digital pulse synthesis technology have injected new vitality into solving the baseline restoration of High-pressure xenon detectors [26].

In 2005, Allen et al. used a third-order Butterworth filter to filter the pulse, which effectively reduced the impact of noise on the energy resolution of the detector, but at the same time, it would reduce the energy resolution without noise [27]. Although it can reduce the vibration and noise sensitivity of the high-pressure xenon detector, there will still be a decrease in energy resolution

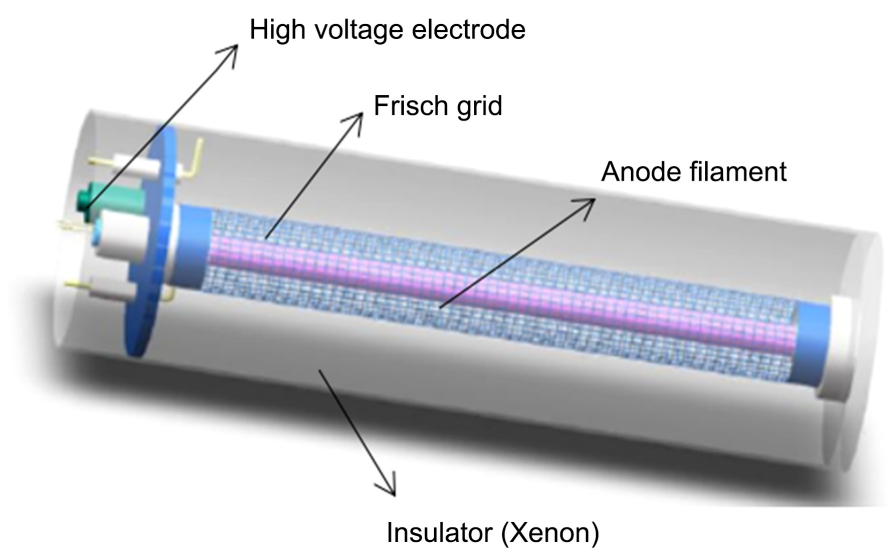

Figure 3. Cylindrical high-pressure xenon detector model [24]. 
under high decibel noise. In 2013, Novikov proposed that external noise vibration will change the capacitance between the gate and the cathode, causing the phase shift of the baseline [28]. In 2014, Detrenko et al. studied that the fundamental reason for the reduction of the energy resolution of high-pressure xenon detectors caused by external noise is the drift of the baseline [29]. At the same time, the digital multi-channel technique of linear interpolation subtracting the baseline is used to restore the baseline, and good results have been achieved. However, this method cannot accurately trigger the arrival of the pulse, and cannot fit the stacked pulse, and the pulse pass rate is low. In 2018, Gao Le et al. introduced mathematical morphology to the correction of digital nuclear pulse baseline drift, designed and implemented a mathematical morphology baseline restoration method that can automatically adjust parameters according to baseline characteristics in real time, and used it on the cadmium zinc telluride detector. The experimental effect is good [11]. However, this mathematical morphology baseline restoration method has not been tested on the high-pressure xenon detector, and its baseline restoration effect is unknown.

\section{Summary and Outlook}

The high-pressure xenon detector has good energy resolution, a wide temperature range and strong anti-radiation characteristics, and has huge application prospects in complex nuclear radiation. At the same time, the high-pressure xenon detector is susceptible to external noise due to its thin grid thickness, causing baseline drift, which reduces or even distorts the energy resolution of the energy spectrum. This article summarizes a large number of documents and finds that the problem cannot be solved fundamentally by changing the structure or material of the high-pressure xenon detector screen grid. With the development of digital signal processing technology, it should be possible to solve the problem of high-pressure xenon detector noise sensitivity through digital baseline restoration algorithms, especially adaptive baseline restoration algorithms. The research on the baseline restoration algorithm of high-pressure xenon detector is carried out in the follow-up work.

\section{Acknowledgements}

Thanks to the teachers and students who participated in this research work.

\section{Conflicts of Interest}

The authors declare no conflicts of interest regarding the publication of this paper.

\section{References}

[1] Xu, K. (2016) Development Trend of Nuclear Radiation Detection Instruments and Technology. Science and Technology Innovation Herald, 13, 68-69.

[2] Cui, X.H., Gu, T.N. and Zhang, Y. (2011) Performance Comparison between Ion 
Implantation Type and Au-Si Surface Barrier Type Semiconductor Detectors. Radiation Protection Communications, 31, 26-28.

[3] Ma, R.G., Zhu, L.H. and Wang, Z.M. (2006) Development of Automatic Liquid Nitrogen Replenishment System for High Purity Germanium Detector. Atomic Energy Science and Technology, No. 1, 92-95.

[4] Gu, Z.W., Yan, W.Q. and Liu, W.B. (2015) Performance Comparison of Lanthanum Bromide, Sodium Iodide and Plastic Scintillation Detectors. Laboratory Research and Exploration, 34, 64-67.

[5] Cao, L., Kang, W. and Hao, F.H. (2010) Research and Application of High-Pressure Xenon $\gamma$ Detector. Nuclear Electronics and Detection Technology, 30, 130-135.

[6] Dmitrenko, V.V. and Chemysheva, I.V. (2000) Vibrostability of High Pressure Xenon Gamma-Ray Detectors. IEEE Transactions on Nuclear Science, 47, 939-943. https://doi.org/10.1109/23.856722

[7] Fan, Y.S., Zhou, Y.P. and Tian, S.H. (2012) Research on Improvement of Multi-Channel Pulse Amplitude Analyzer. Electronic Design Process, 20, 70-72.

[8] Bolotnikov, A.E. and Ramsey, B.D. (1998) Development of High-Pressure Xenon Detectors. Hard X-Ray and Gamma-Ray Detector Physics and Applications, Vol. 3446, 64-76. https://doi.org/10.1117/12.312879

[9] Chen, B.X. and Zhang, Z. (2011) Nuclear Radiation Physics and Detection Science. Harbin Engineering University Press, Harbin.

[10] Gao, L. (2019) High-Pressure Xenon Detector Digital Multi-Channel System and Its Baseline Drift Algorithm Correction. Thesis, Nanjing University of Aeronautics and Astronautics, Nanjing.

[11] Geo, L., Tang, X.B. and Gong, P. (2018) Baseline Restoration Method Based on Mathematical Morphology for High-Pressure Xenon Detectors. Nuclear Instrument and Methods in Physics Research A, 904, 163-170.

https://doi.org/10.1016/j.nima.2018.07.040

[12] Davidson, N. and Larsh, A.E. (1948) Conductivity Pulses in Liquid Argon. Physical Review, 74, 220. https://doi.org/10.1103/PhysRev.74.220

[13] Hutchinson, G.W. (1948) Ionization in Liquid and Solid Argon. Nature, 162, 610. https://doi.org/10.1038/162610a0

[14] Aprile, E., Ku, W.H.M., Park, J., et al. (1987) Energy Resolution Studies of Liquid Argon Ionization Detectors. Nuclear Instruments and Methods in Physics Research Section A, 261, 519-526. https://doi.org/10.1016/0168-9002(87)90362-7

[15] Koeman, H. (1975) Practical Performance of the Transversal Digital Filter in Conjunction with X-Ray Detector and Preamplifier. Nuclear Instruments and Methods, 123, 181-187. https://doi.org/10.1016/0029-554X(75)90093-2

[16] Dmitrienko, V.V., Lebedenko, V.N., Romanyuk, A.S., et al. (1981) Cylindrical Ionization Chamber for Low Energy Gamma Spectrometry (0.1-3 MeV). Pribory $i$ Tekhnika Ehksperimenta, 1981, 49-51.

[17] Mabler, G.J., Yu, B., Smith, G.C., et al. (1997) A Portable Gamma-Ray Spectrometer Using Compressed Xenon. IEEE Nuclear Science Symposium Conference Record, Vol. 1, 769-773.

[18] Tepper, G. and Losee, J. (1995) High Resolution Room Temperature Ionization Chamber Xenon Gamma Radiation Detector. Nuclear Instruments and Methods in Physics Research Section A, 356, 339-346.

https://doi.org/10.1016/0168-9002(94)01206-7 
[19] Levin, C., Germani, J. and Markey, J. (1993) Charge Collection and Energy Resolution Studies in Compressed Xenon Gas near Its Critical Point. Nuclear Instruments and Methods in Physics Research Section, 332, 206-214. https://doi.org/10.1016/0168-9002(93)90760-F

[20] Averin, A.S., Bolotnikov, E.A., Vlasik, F.K., et al. (1990) The High Density Xenon Filled Spectrometer for Cosmic Gamma-Ray Line Observation. International Cosmic Ray Conference, Vol. 4, 150.

[21] Kessick, R. and Tepper, G. (2002) A Hemispherical High-Pressure Xenon Gamma Radiation Spectrometer. Nuclear Instruments and Methods in Physics Research Section A, 490, 243-250. https://doi.org/10.1016/S0168-9002(02)01007-0

[22] Bolotnikov, A., Bolozdynya, A., DeVito, R., et al. (2004) Dual-Anode High-Pressure Xenon Cylindrical Ionization Chamber. IEEE Transactions on Nuclear Science, 51, 1262-1269. https://doi.org/10.1109/TNS.2004.829369

[23] Wang, W., Chen, L. and Wang, H.Y. (2009) Research on the Optimization Design of the Electrode Structure of the Xenon-Charged Ionization Chamber at High Pressure. Nuclear Electronics and Detection Technology Art, 29, 288-293.

[24] Ulin, S.E., Dmitrenko, V.V. and Grachev, V.M. (1995) A Cylindrical Ionization Chamber with a Shielding Mesh Filled with Xenon under a Pressure of 50 Atm. Instruments and Experimental Techniques, 38, 326-330.

[25] Doke, T. (1982) Recent Developments of Liquid Xenon Detectors. Nuclear Instruments and Methods in Physics Research, 196, 87-96. https://doi.org/10.1016/0029-554X(82)90621-8

[26] Abbene, L. and Gerardi, G. (2015) High-Rate Dead-Time Corrections in a General Purpose Digital Pulse Processing System. Journal of Synchrotron Radiation, 22, 1190-1201. https://doi.org/10.1107/S1600577515013776

[27] Seifert, A., Milbrath, B., Pitts, W.K., et al. (2005) Implementation of a Noise Mitigation Strategy for a High-Pressure Xenon Detector. IEEE Nuclear Science Symposium Conference Record, Vol. 3, 1262-1266.

[28] Novikov, A.S., Ulin, S.E. and Dmitrenko, V.V. (2013) New Modification of Xenongamma-Ray Detector with High Energy Resolution. Optical Engineering, 53, Article ID: 021108. https://doi.org/10.1117/1.OE.53.2.021108

[29] Novikov, A.S., Ulin, S.E., et al. (2014) Xenon Detector with High Energy Resolution for Gamma-Ray Line Emission Registration. SPIE 9213, Hard X-Ray, Gamma-Ray, and Neutron Detector Physics, Vol. 9213, Article ID: 921318. https://doi.org/10.1117/12.2060812 